\title{
LÍNEAS ESTRATÉGICAS DE ACCIÓN SOSTENIBLE PARA LOS CENTROS DE ATENCIÓN MÉDICA DE LAS REGIONES DE COLOMBIA
}

\section{Strategic lines of sustainable action for health care centers in Colombia regions}

\author{
Karen Tatiana Valencia Rivero ${ }^{1}$ \\ ${ }^{1}$ Profesora Asociada, Fundación Universitaria Agraria de Colombia, Programa Ingeniería Industrial. Bogotá, \\ Colombia. Investigadora principal, Biomedical Technology for Innovation, Research and Development - BIRD. \\ Bogotá, Colombia. Email: valencia.karen@uniagraria.edu.co
}

(Recibido el 19 de noviembre de 2019 - Aceptado el 19 de mayo de 2020)

\begin{abstract}
Resumen
Con el planteamiento de los Objetivos de Desarrollo Sostenible, la Asamblea General de las Naciones Unidas adoptó un plan de acción para el desarrollo de aspectos sociales, económicos y medioambientales a 2030. Este artículo busca analizar desde una perspectiva cualitativa la sostenibilidad en el campo hospitalario en diferentes partes del mundo donde han implementado estrategias bajo diferentes contextos socio-económicos y culturales que han ayudado a mejorar los procesos eficientemente mientras se reduce el impacto ambiental. La situación actual de la sustentabilidad hospitalaria a nivel global se tomará como punto de partida para proponer estrategias que permitan mejorar los servicios de salud en Colombia a través de la adopción de los métodos más eficientes y exitosos encontrados, que en conjunto con herramientas de la ingeniería industrial y de la ingeniería biomédica permitan abrir nuevos caminos para garantizar una vida saludable desde el cuidado del medio ambiente y de los servicios médicos que se prestan a la sociedad de forma sostenible.
\end{abstract}

Palabras clave: ingeniería industrial, ingeniería biomédica, logística hospitalaria, objetivos de desarrollo, sostenibilidad.

\begin{abstract}
The Sustainable Development Goals defined by the General Assembly of the United Nations, promote an action plan for the development of social, economic and environmental aspects that should be achieved before 2030. This article seeks to analyze from a qualitative perspective sustainable strategies implemented in hospitals at different countries where sustainable strategies were implemented under different socio-economic and cultural contexts, and successfully helped to efficiently improve processes while reducing their environmental impact. Current situation of hospital sustainability at a global level will be taken as a starting point to propose strategies that allow improving health services in Colombia through the adoption of the most efficient and successful methods found, which together with Industrial and Biomedical engineering tools will open new paths to ensure a healthy life.
\end{abstract}

Key words: industrial engineering, biomedical engineering, hospital logistics, sustainable development goals, sustainability.

\section{INTRODUCCIÓN}

Hospitales y Sistemas de Salud a nivel mundial están incorporando prácticas sostenibles en su misión y sus operaciones, a su vez, encuentrando formas de sobresalir, innovar y prosperar en la atención médica [1, 3].
Los de servicios de salud han realizado alianzas y han fortalecido estrategias con proveedores, pacientes y diferentes comunidades, fortaleciéndose mutuamente con la determinación de apoyar un servicio de salud de calidad en un planeta sano [4]. Hoy en día, existen hospitales que son completamente independientes de 
la energía, hospitales que han reinventado el concepto de alimentos hospitalarios saludables al tiempo que respaldan un sistema alimentario sostenible, y hospitales que han utilizado sus voces y su poder de compra para cambiar la forma en que se fabrican los productos para el cuidado de la salud, evitando una gama de productos químicos tóxicos en muebles, productos de limpieza, dispositivos médicos y materiales de construcción, por nombrar algunos [5-7].

A través del análisis de los procesos dentro de las instituciones prestadoras de los servicios médicos se pueden definir criterios de calidad para establecer metas de mejora de procesos haciéndolos sostenibles a través del diseño, aplicación y evaluación de herramientas logísticas. En este artículo se analizan diferentes referentes bibliográficos donde se presentan herramientas sustentables bajo diferentes contextos socio-económicos y culturales, en países como Abu Dhabi [8], Estados Unidos [9,10], Brasil [11], Turquía [12], Polonia [13], Irán [14, 15], Canadá [16], México [17], India [18], Italia [19], Reino Unido [20], Bangladesh [21], y Kenia [22].

En el marco de la disponibilidad y accesibilidad de servicios integrales de salud en centros médicos en el país, el mejoramiento en la logística para el acceso a los servicios integrales de salud, la adopción de estrategias de gestión ambiental, la protección de los recursos naturales y humanos, el control de la contaminación, y el fomento de un cambio cultural para pasar de un consumo excesivo de recursos a un uso de energía y elementos cada vez menor o más racional, hacen que esta clase de propuestas tengan un alto impacto a nivel socio-económico, especialmente, donde la demanda de salud tiene los niveles más bajos de atención. A continuación, se categorizan las áreas de los hospitales susceptibles de modificar sus procesos por unos más sostenibles y se analiza su situación actual en los diferentes centros médicos.

\section{SOSTENIBILIDAD HOSPITALARIA}

En general, los pequeños proveedores de salud en las comunidades rurales, tienen un acceso limitado a recursos laborales y de capital en comparación con sus homólogos más grandes; sin embargo, ellos enfrentan las mismas presiones regulatorias de la acreditación hospitalaria con respecto a la calidad de la atención al paciente y a la seguridad. Los hospitales y centros de salud pequeños pueden implementar estrategias a nivel empresarial que no solo creen un entorno de atención y seguridad de calidad, sino que también establezcan las bases para mejorar el control de costos mientras se siguen estrategias sostenibles que reduzcan el impacto ambiental y social de sus procesos [9].

Metodologías como la Lean que fueron inventadas por el Sistema de producción de Toyota (TPS) para superar las ineficiencias comerciales, han intervenido nuevos escenarios como el de la salud buscando responder a las necesidades de pequeñas y grandes empresas de distintos sectores [9]. El concepto Lean de minimización de residuos para la mejora de procesos se ha implementado recientemente en servicios como la atención médica, que ha adoptado este tipo de gestión para el desarrollo de un programa de mejora de procesos $[23,24]$. Independientemente de la estrategia logística, de gestión, o de operaciones a utilizar, se espera que estas estrategias por lo menos respondan a La Triple Línea de Fondo que se ha definido internacionalmente y que es conocida como una integración de tres dimensiones de sostenibilidad: económica, ambiental y social, promoviendo una responsabilidad social corporativa [25, 26].

\section{PROPUESTA DE SOSTENIBILIDAD HOSPITALARIA PARA LAS REGIONES DE COLOMBIA}

Se propone implementar la Triple Línea de Fondo con el concepto lean para la minimización de residuos y mejora de procesos como el transporte, inventario, movimiento, tiempos de espera, sobre producción, y sobre procesamiento [27]; así mismo, se busca promover la satisfacción de los empleados y de los pacientes con la disponibilidad de citas, la calidad de la atención y la garantía de los servicios básicos, mejorando los procesos con herramientas logísticas y de gestión [28]. 
Con el Sistema de producción de Toyota (TPS), se puede especificar cómo se realiza el trabajo, cómo se transfiere el conocimiento entre los trabajadores y dentro del sistema, cómo se coordina la producción entre tareas y servicios, y cómo es el proceso controlado, medido y sostenido. Se propone el uso de reglas que también prescriben implícitamente la estructura organizacional y los sistemas de control que se requieren para implementar TPS de manera rentable y sostenible.

Se especifican a continuación cuatro reglas que forman la base de TPS como una aplicación adecuada dentro de los centros médicos: (i) Todo el trabajo debe estar altamente especificado en cuanto a contenido, secuencia, tiempo y resultado. (ii) Cada conexión cliente-proveedor debe ser directa, y debe haber una forma inequívoca de sí o no para enviar solicitudes y recibir respuestas. (iii) El camino para cada producto y servicio debe ser simple y directo. (iv) Cualquier mejora debe hacerse de acuerdo con el método científico, bajo la guía de un maestro, en el nivel más bajo posible de la organización [9].

Observaciones de los procesos en los centros de salud como se realizan en la actualidad, deben ejecutarse para, posteriormente, plantear estrategias entorno a oportunidades de mejora identificados. El desarrollo de herramientas tangibles para la resolución de problemas también puede incluirse en la ejecución de estrategias para el mejoramiento de procesos y su calidad. Dispositivos biomédicos pueden nacer de la identificación de necesidades, en este caso en el entorno hospitalario, al mismo tiempo, que la implementación de estrategias logísticas y de gestión se muestran necesarias en los procesos. La calidad de la observación y análisis de los procesos debe garantizar la correcta identificación de oportunidades de mejora de procesos y de creación de dispositivos biomédicos.

Respecto a la gestión de la cadena de suministro del servicio, este se debe basar en tres procesos (i) la gestión de procesos, (ii) la gestión de componentes, y (iii) la configuración de la red, lo que implica que los conceptos deben extraerse sobre la base de estos procesos
[15]. Adicionalmente, la información presentada con antelación, se plantean una serie de líneas estratégicas para observar en detalle y mejorar los procesos desde la sostenibilidad en los centros de atención de salud de las regiones de Colombia.

\subsection{Huella Hídrica}

La conservación y eficiencia en el uso del agua es fundamental. Se debe lograr el consumo de menos galones de agua por pie cuadrado, plantear proyectos de conservación y reducción del consumo de agua con el tiempo a través de la innovación.

\subsection{Huella de Carbono}

Se debe trabajar por el seguimiento y la medición de las emisiones de $\mathrm{CO} 2$ y el desarrollo general de un programa climático. Los hospitales deben comprender los impactos del cambio climático en la salud y tomar medidas para mitigar esos impactos, principalmente, a través de la educación de su personal y de las comunidades para que desempeñen un papel de apoyo.

\subsection{Eficiencia Energética}

Se debe procurar una eficiencia energética y una planificación estratégica del uso de energía. Son necesarios administradores de energía y programas escritos para reducir el uso de energía con el tiempo. Se debe rastrear la intensidad del uso de energía, y procurar la transición a fuentes de energía renovables.

\subsection{Manejo de Residuos}

El manejo adecuado de materiales de residuo se debe demostrar a través de altas tasas de reciclaje, baja generación de desechos médicos regulados, y un claro enfoque en minimizar el volumen total de desechos generados por las instituciones.

\subsection{Manejo de Residuos Químicos}

Se deben propender políticas sólidas de reducción de químicos. Se propone abordar la toxicidad a través de programas de limpieza más ecológicos donde también se evite al máximo el uso de productos químicos perjudiciales, y se muestre interés por comprar productos, servicios 
y equipos sostenibles. Así también, se debe eliminar el uso del mercurio y priorizar la capacitación del personal que maneja los productos químicos peligrosos para evitar accidentes y contaminaciones.

\subsection{Alimentación Saludable de Pacientes}

En el ámbito de los alimentos, se deben buscar estrategias como la reducción del consumo de carne, la adquisición de una mejor carne, el abastecimiento local y sostenible para la alimentación dentro de los hospitales, mejorando el acceso al agua del grifo y bebidas saludables, apoyando el acceso a alimentos saludables y evitando desechos alimentarios. También son necesarias políticas sólidas y estrategias educativas que aborden el sistema alimentario como un factor crítico dentro de un plan general de sostenibilidad para la salud humana.

\subsection{Compras Ambientalmente Preferibles}

Las políticas de compra, interacciones con proveedores, contratos ambientalmente preferibles son estrategias para reducir el impacto negativo de los prestadores de servicios de salud [10].

\subsection{Construcciones Verdes}

Aunque muchos de estos centros no están pensando en ampliar su infraestructura, se puede procurar con cierta proyección ambiental la ampliación o construcción de nuevas sedes, así como las renovaciones importantes, idealmente hacia la certificación LEED (Leadership in Energy \& Environmental Design), y otros productos ecológicos innovadores. Este tipo de construcciones incluyen eficiencia energética y de agua, materiales más seguros, abastecimiento regional, integración de la naturaleza y entre otros mecanismos.

\section{DISCUSIÓN}

Las estrategias aquí propuestas no están exentas de ser afectadas por barreras que surjan en la implementación. Por nombrar algunas: la falta de actitud y compromiso al cambio de las personas involucradas en los procesos [29], falta de comprensión de la propuesta en los diferentes niveles organizacionales [30], falta de recursos y limitaciones presupuestarias [31], un vínculo débil entre los programas de mejora y el nivel estratégico de la organización [32], así como, la falta de estrategias de mejora al largo plazo [31].

Adicionalmente, el sector de la salud es diferente de otros sectores debido a su complejidad dada por los dos aspectos profesionales diferentes que se encuentran en este sector: los profesionales médicos y los no médicos. Los profesionales médicos están familiarizados con los términos y conocimientos propios de su disciplina, mientras que los profesionales no médicos están tan calificados como los profesionales médicos, pero no disponen del conocimiento de conceptos médicos, aunque están debidamente capacitados para gestionar diferentes funciones de organización, y trabajan con una perspectiva diferente [12].

Esta perspectiva bilateral puede ser vista como un problema, pero en este artículo se propone como una oportunidad que de ser bien mediada por profesionales que entiendan ambos campos, como los ingenieros biomédicos, puede resultar en excelentes estrategias que integren interdisciplinarmente herramientas para la solución de problemas en el sector salud. Es necesaria la implementación de las estrategias aquí propuestas en pequeños centros de salud de diferentes regiones de Colombia para ampliar la visión del estudio y profundizar en las necesidades específicas de la población Nacional.

\section{CONCLUSIÓN}

Atendiendo al Consejo Empresarial Mundial para el Desarrollo Sostenible, es indispensable que las empresas, incluyendo las relacionadas con el sector salud, se comporten éticamente contribuyendo al desarrollo económico al tiempo que se mejora la calidad de vida de la fuerza laboral y sus familias, de la comunidad local, y de la sociedad en general. En este artículo, se propone el alcance de este objetivo a través de la vinculación de estrategias sostenibles en los centros de salud, promoviendo así no sólo un trabajo firme en pro del alcance de los objetivos de desarrollo sostenible, sino del 
desarrollo en general de la sociedad colombiana.

\section{REFERENCIAS}

[1] Silvius AG, Schipper R. A maturity model for integrating sustainability in projects and project management. 24th World Congress of the International Project Management Association (IPMA) Istanbul, Turkey; 2010.

[2] Visser WAM. Sustainability reporting in South Africa. Corp Environ Strat. 2002;9(1):79-85.

[3] Holliday C. Sustainable growth, the DuPont way. Harv Bus Rev. 2001;79(8):129-132.

[4] Marimuthu M, Paulose $H$. Emergence of sustainability-based approaches in healthcare: expanding research and practice. Procedia-Soc Behav Sci. 2016;224:554-561.

[5] WCED. World Commission on Environment and Development. Our common future. Oxford: Oxford University Press; 1987.

[6] Deloitte \& Touche. Business strategy for sustainable development: leadership and accountability for the 90s. IISD; 1992.

[7] Elkington J. Cannibals with forks: the triple-bottom line for 21st century business. Canada: New Society Publishers; 1998.

[8] Omar Awad AlJaberi, Matloub Hussain \& Paul R. Drake (2017): A framework for measuring sustainability in healthcare systems, International Journal of Healthcare Management, DOI: 10.1080/20479700.2017.1404710

[9] Kevin F. Collins \& Senthil K. Muthusamy (2007) Applying the Toyota Production System to a Healthcare Organization: A Case Study on a Rural Community Healthcare Provider, Quality Management Journal, 14:4, 41-52, DOI: 10.1080/10686967.2007.11918045

[10] Thomas M. Schieble MD, MBA (2008) Advertised Sustainability Practices Among Suppliers to a University Hospital Operating Room, Journal of Hospital Marketing \& PublicRelations, 18:2, 135148, DOI: $10.1080 / 15390940802232424$

[11] Higor Leite, Nicola Bateman \& Zoe Radnor (2019):
Beyond the ostensible: an exploration of barriers to lean implementation and sustainability in healthcare, Production Planning \& Control, DOI: 10.1080/09537287.2019.1623426

[12] Songul Cinaroglu (2016) Complexity in healthcare management: Why does Drucker describe healthcare organizations as a doubleheaded monster? International Journal of Healthcare Management, 9:1, 11-17, DOI: 10.1179/2047971915Y.0000000016

[13] Justyna Przychodzen \& Wojciech Przychodzen (2013) Corporate sustainability and shareholder wealth, Journal of Environmental Planning and Management, 56:4, 474-493, DOI: 10.1080/09640568.2012.685927

[14] Yusef Shaabani, Ali Vafaee Najar, Mohammad Naser Shafiee, Marziyhe Meraji \& Elaheh Hooshmand (2019): Designing a green hospital model: Iranian hospital, International Journal of Healthcare Management, DOI: 10.1080/20479700.2019.1572265

[15] Seyed Habibollah Mirghafoori1, Ali Morovat Sharifabadi1, Salim Karimi Takalo (2018) Development of Causal Model of Sustainable Hospital Supply Chain Management Using the Intuitionistic Fuzzy Cognitive Map (IFCM) Method, Journal of Industrial Engineering and Management, https://doi.org/10.3926/jiem.2517

[16] Jennifer Y. Verma, Meghan Rossiter, Kirby Kirvan, Jean-Louis Denis, Stephen Samis, Kaye Phillips, Kim Venu, Donna Allen, G. Ross Baker, Mireille Brosseau, François Champagne, Catherine Gaulton, Erin Leith \& Patty O'connor (2013) Going far together: Healthcare collaborations for innovation and improvement in Canada, International Journal of Healthcare Management, 6:2, 66-76, DOI: 10.1179/2047971913Y.0000000029

[17] Gabriela Citlalli López-Torres, Jose Arturo GarzaReyes, Gonzalo Maldonado-Guzmán, Vikas Kumar Luis Rocha-Lona \& Anass Cherrafi (2019) Knowledge management for sustainability in operations, Production Planning \& Control, 30:10-12, 813-826, DOI: 10.1080/09537287.2019.1582091 
[18] Sirish Kumar Gouda \& Haritha Saranga (2018) Sustainable supply chains for supply chain sustainability: impact of sustainability efforts on supply chain risk, International Journal of Production Research, 56:17, 5820-5835, DOI: 10.1080/00207543.2018.1456695

[19] Silvia Barbero \& Agnese Pallaro (2017) Systemic Design for Sustainable Healthcare, The Design Journal, 20:sup1, S2473-S2485, DOI: 10.1080/14606925.2017.1352762

[20] Maria K. Triantafyllou \& Tom J. Cherrett (2010) The logistics of managing hazardous waste: a case study analysis in the UK retail sector, International Journal of Logistics: Research and Applications, 13:5, 373394, DOI: 10.1080/13675567.2010.512150

[21] Toufiq Ahmed \& Kunio Shirahada (2019) Toward a sustainable healthcare service system in a limited resource context: Case study of Bangladesh rural advancement committee's healthcare system, International Journal of Healthcare Management, 12:2, 97-105, DOI: 10.1080/20479700.2017.1389512

[22] W. Leal Filho, S. K. Tripathi, J. B. S. O. D. Andrade Guerra, R. Giné-Garriga, V. Orlovic Lovren \& J. Willats (2019) Using the sustainable development goals towards a better understanding of sustainability challenges, International Journal of Sustainable Development \& World Ecology, 26:2, 179-190, DOI: 10.1080/13504509.2018.1505674

[23] Hussain M, Khan M, Al-Aomar R. A framework for supply chain sustainability in service industry with confirmatory factor analysis. Renew Sustain Energy Rev. 2016;55:1301-1312.

[24] Hussain M, Malik M, Al Neyadi HS. AHP framework to assist lean deployment in Abu Dhabi public healthcare delivery system. Bus Process Manag J. 2016;22 (3):546-565.

[25] Elkington J. Cannibals with forks: the triple-bottom line for 21st century business. Canada: New Society Publishers; 1998.

[26] Kotler P, Lee N. Corporate social responsibility: doing the most good for your company and your cause; 2006.
[27] NHSIII. Going lean in the NHS, NHS intuitive for innovation and improvement, Warwick; 2007. (cited 2014 Jul 14). Available from www. northamptongeneral.nhs.uk/Downloads/ OurServices/Servicelmprovement/ Toolsforimprovement/GoingleanintheNHS.pdf

[28] Ramsaran-Fowdar RR. Identifying health care quality attributes. J Health Hum Serv Adm. 2005: 428-443.

[29] Kinder, T., and T. Burgoyne. 2013. "Information Processing and the Challenges Facing Lean Healthcare." Financial Accountability and Management 29(3): 271-290. doi:10.1111/ faam.12016.

[30] Andersen, H., K. A. Røvik, and T. Ingebrigtsen. 2014. "Lean Thinking in Hospitals: is There a Cure for the Absence of Evidence? A Systematic Review of Reviews." BMJ Open 4(1): e003873. doi:10.1136/ bmjopen-2013-003873.

[31] Albliwi, S., J. Antony, S. Abdul Halim Lim, and T. Van der Wiele. 2014. "Critical Failure Factors of Lean Six Sigma: A Systematic Literature Review." International Journal of Quality and Reliability Management 31(9): 1012-1030. doi:10.1108/ IJQRM-09-2013-0147.

[32] Bhamu, J., and K. Singh Sangwan. 2014. "Lean Manufacturing: literature Review and Research Issues." International Journal of Operations and Production Management 34(7): 876-940. doi:10.1108/IJOPM-08-2012-0315. 